\title{
Quasicausal expansion of the quantum Liouville propagator
}

\author{
G. W. Bund* \\ Instituto de Física Teórica, Universidade Estadual Paulista, rua Pamplona 45, São Paulo, São Paulo 01405-900, Brazil \\ S. S. Mizrahi ${ }^{\dagger}$ \\ Departamento de Física, Universidade Federal de São Carlos, Rodovia Washington Luiz Km 235,13565-905 \\ São Carlos, São Paulo, Brazil \\ M. C. Tijero \\ Instituto de Física, Universidade Estadual Paulista and Pontifícia Universidade Católica (PUC), São Paulo, São Paulo, Brazil
}

(Received 14 July 1995)

\begin{abstract}
The quasicausal expansion of the quantum Liouville propagator is introduced into the Weyl-Wigner picture. The zeroth-order term is shown to lead to the statistical quasiclassical method of Lee and Scully [J. Chem. Phys. 73, 2238 (1980)].

PACS number(s): $34.10 .+\mathrm{x}, 03.65 . \mathrm{Sq}$
\end{abstract}

\section{INTRODUCTION}

Several works were devoted to the development of semiclassical methods in order to describe collision phenomena in the molecular, atomic, and nuclear heavy-ion domains [1-3]. Among these, some have adopted the quantum WeylWigner (WW) picture [4,5], since it possesses a well suited structure that enables the development of $\hbar$ power-series expansions. In Refs. [4,6] the reader can find a detailed discussion about the problems and difficulties involving these expansions. Lee and Scully [2] have developed a method, first suggested by Heller [7], that they called the statistical quasiclassical method and they applied it to calculate transition probabilities of colinear molecular collisions. However, some of their calculated transitions present discrepancies with the exact quantum-mechanical values [8]. The aim of this paper is to show that the approach of Lee and Scully corresponds to the zeroth order of a general expansion of the full quantum Liouvillian written in the WW picture; therefore their calculated transition probabilities accept higherorder corrections, and here we derive the analytical expression of the first-order term.

As will be made clear later, we find it more suitable to call the zeroth-order term the causal approximation (CA) and the first-order term as the quasicausal approximation (QCA). But first it will be necessary to clarify the meaning of taking the classical limit, which in general leads to annoying singularities [7] in the Wigner distribution function (WDF). We understand that Planck's constant arises in the quantum Liouville equation from two sources: one of these being the WDF at initial time and the other the presence of $\hbar$ in the quantum evolution operator $\exp \left[-i \mathscr{C}_{Q}\left(t-t_{0}\right)\right]$, through the Liouville operator $\mathscr{L}_{Q}$. In the next section we show how to handle the limit $\hbar \rightarrow 0$.

\footnotetext{
*Electronic address: bund@vax.ift.unesp.br ${ }^{\dagger}$ Electronic address: dsmi@ power.ufscar.br

\#Electronic address: maria@vax.ift.unesp.br
}

\section{CAUSAL AND QUASICAUSAL APPROXIMATIONS}

Let us consider the quantum Liouville equation in the WW picture [4],

$$
\frac{\partial W(\vec{p}, \vec{q}, t)}{\partial t}=-i \mathscr{B}_{Q} W(\vec{p}, \vec{q}, t),
$$

where $W(\vec{p}, \vec{q}, t)$ is the WDF and $(\vec{p}, \vec{q})$ is a point of phase space in one or several degrees of freedom. The quantum Liouvillian is

$$
\mathscr{L}_{Q}=H(\vec{p}, \vec{q})\left[i \frac{2}{\hbar} \sin \frac{\hbar}{2} \overleftrightarrow{\Lambda}\right],
$$

$H(\vec{p}, \vec{q})$ being the Hamiltonian of the system.

The operator

$$
\overleftrightarrow{\Lambda}=\frac{\overleftarrow{\partial}}{\partial \vec{q}} \cdot \frac{\vec{\partial}}{\partial \vec{p}}-\frac{\overleftarrow{\partial}}{\partial \vec{p}} \cdot \frac{\vec{\partial}}{\partial \vec{q}}
$$

is the Poisson bracket, where the arrows indicate on which side the derivatives operate.

In order to develop a $\hbar$ power-series expansion and to analyze the classical limit, we introduce the dimensionless parameter $\alpha$ in $\mathscr{L}_{Q}$,

$$
\mathscr{L}_{Q}^{(\alpha)}=H(\vec{p}, \vec{q})\left[i \frac{2}{\alpha \hbar} \sin \frac{\alpha \hbar}{2} \overleftrightarrow{\Lambda}\right]
$$

The formal solution of Eq. (1) is given by

$$
W^{(\alpha)}(\vec{p}, \vec{q}, t)=e^{-i \mathscr{L}_{Q}^{(\alpha)}\left(t-t_{0}\right)} W_{0}(\vec{p}, \vec{q}),
$$

where $W_{0}(\vec{p}, \vec{q})$ is the WDF at the initial time $t_{0}$. In the limit $\alpha \rightarrow 0, \mathscr{L}_{Q}^{(\alpha)}$ goes over into the classical Liouvillian

$$
\mathscr{L}_{c l}=i H(\vec{p}, \vec{q}) \overleftrightarrow{\Lambda}
$$

which becomes $\hbar$ independent, and Eq. (5) is written as [9] 


$$
\begin{aligned}
W^{(0)}(\vec{p}, \vec{q}, t) & =e^{-i \mathscr{L}_{c l}\left(t-t_{0}\right)} W_{0}(\vec{p}, \vec{q}) \\
& =W_{0}\left(\vec{p}\left(t_{0}-t\right), \vec{q}\left(t_{0}-t\right)\right) .
\end{aligned}
$$

Each point of the phase space of the initial WDF will evolve classically (although it will be reversed in time) following a trajectory according to Hamilton equations; however, quantum ingredients may still be present through the choice of $W_{0}(\vec{p}, \vec{q})$ at time $t_{0}$. This description constitutes the CA and it is the same approximation originally adopted in Ref. [2]. It is important to realize that the introduction of the parameter $\alpha$ avoids the confusion between the different origins of $\hbar$ in the WDF when we consider the limit $\hbar \rightarrow 0$. Thus, to obtain the CA this limit has to be considered in the Liouvillian only and not in the initial time WDF, where quantum ingredients are present and must remain. In order to get the first-order approximation we have to perform, initially, the expansion of $\mathscr{L}_{Q}^{(\alpha)}$ in a power series of $(\alpha \hbar)^{2}$,

$$
\mathscr{L}_{Q}^{(\alpha)}=\sum_{n=0}^{\infty}(\alpha \hbar)^{2 n} \mathscr{L}_{2 n}
$$

where

$$
\mathscr{L}_{2 n}=H(\vec{p}, \vec{q})\left[i \frac{(-1)^{n}}{2^{2 n}(2 n+1) !}(\overleftrightarrow{\Lambda})^{2 n+1}\right]
$$

(note that $\mathscr{L}_{0}=\mathscr{L}_{c l}$ ), which permits us to write Eq. (1) as

$$
\begin{aligned}
& \frac{\partial W^{(\alpha)}(\vec{p}, \vec{q}, t)}{\partial t}+i \mathscr{C}_{0} W^{(\alpha)}(\vec{p}, \vec{q}, t) \\
& \quad=-i \sum_{n=1}^{\infty}(\alpha \hbar)^{2 n} \mathscr{L}_{2 n} W^{(\alpha)}(\vec{p}, \vec{q}, t) .
\end{aligned}
$$

Using the classical propagator $e^{-i \mathscr{L}_{0}\left(t-t_{0}\right)}$, the integral equation corresponding to Eq. (10) is

$$
\begin{aligned}
W^{(\alpha)}(\vec{p}, \vec{q}, t)= & e^{-i \mathscr{L}_{0}\left(t-t_{0}\right)} W_{0}(\vec{p}, \vec{q})-i \sum_{n=1}^{\infty}(\alpha \hbar)^{2 n} \\
& \times \int_{t_{0}}^{t} d t^{\prime} e^{-i \mathscr{L}_{0}\left(t-t^{\prime}\right)} \mathscr{L}_{2 n} W^{(\alpha)}\left(\vec{p}, \vec{q}, t^{\prime}\right) .
\end{aligned}
$$

This equation may be solved iteratively to any order in $(\alpha \hbar)^{2}$; at first order we establish the QCA

$$
\begin{aligned}
W_{Q C A}^{(\alpha)}(\vec{p}, \vec{q}, t)= & e^{-i \mathscr{L}_{0}\left(t-t_{0}\right)} W_{0}(\vec{p}, \vec{q})-i(\alpha \hbar)^{2} \\
& \times \int_{t_{0}}^{t} d t^{\prime} e^{-i \mathscr{L}_{0}\left(t-t^{\prime}\right)} \mathscr{D}_{2} e^{-i \mathscr{L}_{0}\left(t^{\prime}-t_{0}\right)} W_{0}(\vec{p}, \vec{q}) .
\end{aligned}
$$

It can be verified that for $\alpha=0$ the CA is obtained, but for $\alpha \neq 0$ the terms on the right-hand side of Eq. (10) are responsible for the breaking of the causal evolution of the WDF phase-space points. Moreover, it should be noted that in Eq. (12) the only evolution operator connecting different times is $e^{-i \mathscr{L}_{0}\left(t-t_{0}\right)}$, which gives a causal character to the evolution. If we wish to analyze the behavior of the classical limit we first have to make $\alpha=0$ and thereafter $\hbar \rightarrow 0$ in $W_{Q C A}^{(\alpha)}(\vec{p}, \vec{q}, t)$, since the operator $\mathscr{L}_{2}$ may still bring down $\hbar^{-1}$ factors. If such a limit exists the classical distribution function $\delta(\vec{p}-\vec{P}(t)) \delta(\vec{q}-\vec{Q}(t))$ is to be obtained, where $\vec{P}(t)$ and $\vec{Q}(t)$ are the solutions of Hamilton equations. Otherwise, if the classical limit is of no interest, it is sufficient to take $\alpha=1$ after Eq. (11) has been iterated up to the desired order in $(\alpha \hbar)^{2}$.

\section{TRANSITION PROBABILITIES FOR A COLLISIONAL PROCESS}

We are now going to derive the expression of the transition probabilities up to the QCA, corresponding to the collisional process treated in Refs. [2] and [8], where a molecule with a known internal structure suffers a collision from a pointlike projectile; the molecule is transferred from the initial discrete energy level $|m\rangle$ to the final level $|n\rangle$. The density matrix for the target plus projectile system is $\rho\left(\vec{R}, \vec{R}^{\prime} ; \vec{x}, \vec{x}^{\prime}, t\right)=\left\langle\vec{R}, \vec{x}|\hat{\rho}(t)| \vec{R}^{\prime}, \vec{x}^{\prime}\right\rangle$, where the coordinates $\vec{R}, \vec{R}^{\prime}$ specify the relative distance between the projectile and the $c . m$. of the target, while $\vec{x}, \vec{x}^{\prime}$ refer to one or more internal coordinates of the target. The density matrix is related to the WDF by [1]

$$
\begin{aligned}
\rho\left(\vec{R}, \vec{R}^{\prime} ; \vec{x}, \vec{x}^{\prime}, t\right)= & \int d \vec{P} d \vec{p} e^{i \frac{i}{\hbar} \cdot\left(\vec{R}-\vec{R}^{\prime}\right)} e^{i \hbar^{\vec{p} \cdot\left(\vec{x}-\vec{x}^{\prime}\right)}} \\
& \times W\left(\vec{P}, \frac{1}{2}\left(\vec{R}+\vec{R}^{\prime}\right), \vec{p}, \frac{1}{2}\left(\vec{x}+\vec{x}^{\prime}\right), t\right),
\end{aligned}
$$

and the matrix element that is appropriate to a final state $|n\rangle$ then becomes

$$
\begin{aligned}
\rho_{n}(\vec{R}, t) & =\langle n \vec{R}|\hat{\rho}(t)| n \vec{R}\rangle \\
& =\int d \vec{x} d \vec{x}^{\prime} \varphi_{n}^{*}(\vec{x}) \varphi_{n}\left(\vec{x}^{\prime}\right) \rho\left(\vec{R}, \vec{R}^{\prime} ; \vec{x}, \vec{x}^{\prime}, t\right),
\end{aligned}
$$

where the $\varphi_{n}(\vec{x})$ 's are the eigenfunctions of the Hamiltonian for the internal degrees of freedom. Introducing the WDF $W_{n}$ corresponding to the internal motion, defined through

$$
\varphi_{n}^{*}(\vec{x}) \varphi_{n}\left(\vec{x}^{\prime}\right)=\int d \vec{p}^{\prime} e^{-\frac{i}{\hbar} \vec{p}^{\prime} \cdot\left(\vec{x}-\vec{x}^{\prime}\right)} W_{n}\left(\vec{p}^{\prime}, \frac{1}{2}\left(\vec{x}+\vec{x}^{\prime}\right)\right)
$$

into Eq. (14), making further the change of variables $\vec{\xi}=\vec{x}-\vec{x}, \vec{q}=\frac{1}{2}\left(\vec{x}+\vec{x}^{\prime}\right)$ and integrating over $\vec{\xi}$ and $\vec{p}^{\prime}$ in this order, we obtain

$$
\begin{aligned}
\rho_{n}(\vec{R}, t)= & (2 \pi \hbar)^{3} \int d \vec{P} d \vec{p} d \vec{q} W(\vec{P}, \vec{R} ; \vec{p}, \vec{q}, t) W_{n}(\vec{p}, \vec{q}) \\
= & (2 \pi \hbar)^{3} \int d \vec{P} d \vec{p} d \vec{q} W_{n}(\vec{p}, \vec{q}) e^{-i \mathscr{C}_{Q}\left(t-t_{0}\right)} \\
& \times W_{0}(\vec{P}, \vec{R} ; \vec{p}, \vec{q})
\end{aligned}
$$


$\mathscr{L}_{Q}$ being the complete Liouvillian of the system and $W_{0}(\vec{P}, \vec{R}, \vec{p}, \vec{q})$ the WDF at time $t_{0}$. The total transition probability is then for $t \rightarrow \infty$ and $t_{0} \rightarrow-\infty$

$$
P_{m \rightarrow n}=\int d \vec{R} d \vec{P} d \vec{p} d \vec{q} W_{0}(\vec{P}, \vec{R} ; \vec{p}, \vec{q}) e^{i \mathscr{L} Q^{\left(t-t_{0}\right)}} W_{m}(\vec{p}, \vec{q})
$$

where the unitarity property of the evolution operator was utilized. The WDF at time $t_{0}$ is chosen such that the projectile is at position $\vec{R}_{0}\left(R_{0} \rightarrow \infty\right.$ or outside the interaction range), while the target is in the state $|m\rangle$. As no initial correlations between target and projectile are assumed we write

$$
W_{0}(\vec{P}, \vec{R} ; \vec{p}, \vec{q})=\delta\left(\vec{R}-\vec{R}_{0}\right) \delta\left(\vec{P}-\vec{P}_{0}\right) W_{m}(\vec{p}, \vec{q}),
$$

which substituted into Eq. (17) yields for $t \rightarrow \infty$ and $t_{0} \rightarrow-\infty$

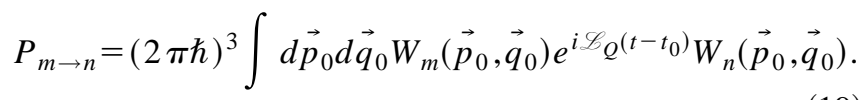

Up to this point the above result is exact, and now, on the basis of what was discussed in Sec. II, we introduce the QCA that consists of replacing the exact by the quasicausal propagator, and this leads in the limit for $t \rightarrow \infty$ and $t_{0} \rightarrow-\infty$ to

$$
\begin{aligned}
P_{m \rightarrow n}^{Q C A}= & (2 \pi \hbar)^{3}\left\{\int d \vec{p}_{0} d \vec{q}_{0} W_{m}\left(\vec{p}_{0}, \vec{q}_{0}\right) W_{n}(\vec{p}(t), \vec{q}(t))\right. \\
& +i(\alpha \hbar)^{2} \int d \vec{p}_{0} d \vec{q}_{0} W_{m}\left(\vec{p}_{0}, \vec{q}_{0}\right) \int_{t_{0}}^{t} d t^{\prime} e^{i \mathscr{L}_{0}\left(t-t^{\prime}\right)} \mathscr{L}_{2} \\
& \left.\times W_{n}\left(\vec{p}\left(t^{\prime}-t_{0}\right), \vec{q}\left(t^{\prime}-t_{0}\right)\right)\right\}
\end{aligned}
$$

where $\vec{p}(t), \vec{q}(t), \vec{p}\left(t-t^{\prime}\right), \vec{q}\left(t-t^{\prime}\right)$ depend on the initial values $\vec{p}_{0}, \vec{q}_{0}, \vec{R}_{0}, \vec{P}_{0}$ but nevertheless $P_{m \rightarrow n}^{Q C A}$ shall be independent on the coordinate $\vec{R}_{0}$. The first term in Eq. (20) corresponds to the $\mathrm{CA}$, while the second term characterizes the QCA. Quantitative evaluations of $P_{m \rightarrow n}^{Q C A}$ have been carried out [10], and they will be presented in due course.

\section{ACKNOWLEDGMENT}

S.S. Mizrahi thanks CNPq, Brazil, for partial financial support.
[1] P. Carruthers and F. Zachariasen, Rev. Mod. Phys. 55, 245 (1983), and references therein.

[2] H. W. Lee and M. O. Scully, J. Chem. Phys. 73, 2238 (1980).

[3] H. Esbensen, Nuclear Structure and Heavy Ion Collisions, Proceedings of the International School of Physics "Enrico Fermi," Course LXXVII, Varenna on Lake Como, 1979, edited by R. A. Broglia, R. A. Ricci, and C. H. Dasso (North-Holland, Amsterdam, 1981), pp. 572-591.

[4] S. R. de Groot and L. G. Suttorp, in Foundations of Electrodynamics (North-Holland, Amsterdam, 1972), Chap. VI.
[5] M. Gadella, Fortschr. Phys. 43, 229 (1995).

[6] G. W. Bund, J. Phys. A 28, 3709 (1995).

[7] E. J. Heller, J. Chem. Phys. 65, 1289 (1976).

[8] D. Secrest and B. R. Johnson, J. Chem. Phys. 45, 4556 (1966).

[9] D. N. Zubarev, in Nonequilibrium Statistical Thermodynamics, Studies in Soviet Science (Consultants Bureau, New York, 1974).

[10] M. C. Tijero, Ph.D. thesis, Instituto de Física Teorica, 1994 (unpublished). 